
$A=\ldots$
11332078

\title{
THERMIONIC SCREENING OF BODIES IN ATMOSPHERE \\ AND INTERPLANETARY SPACE
}

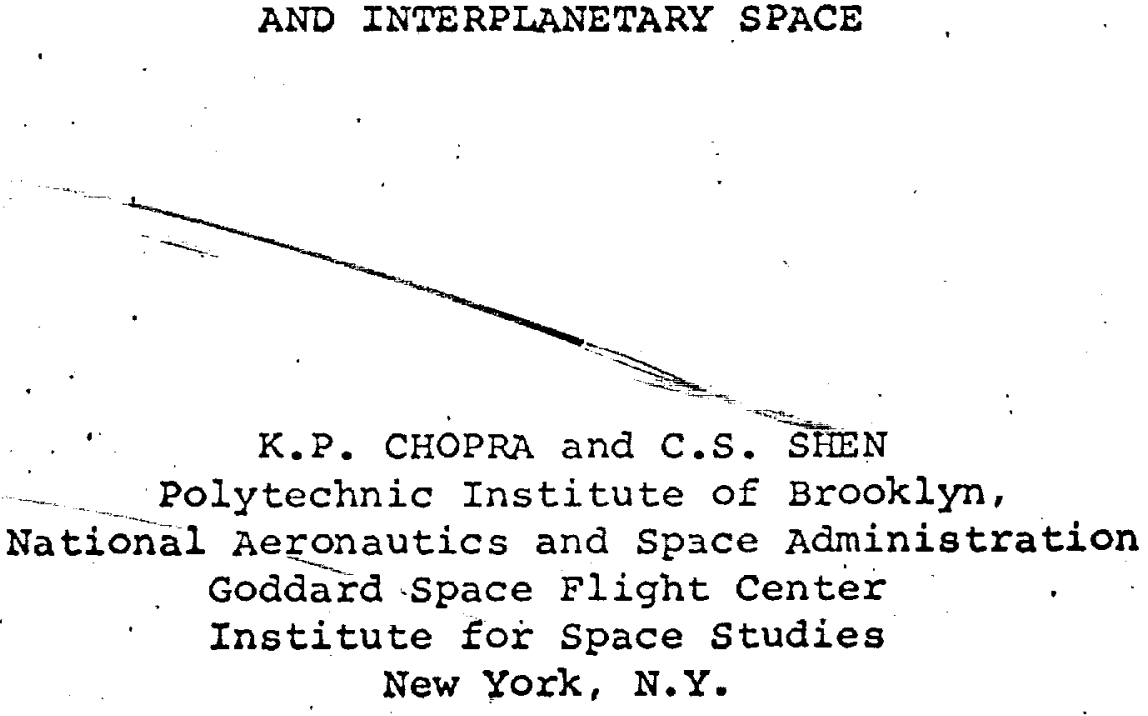

Received April 5, 1963

3

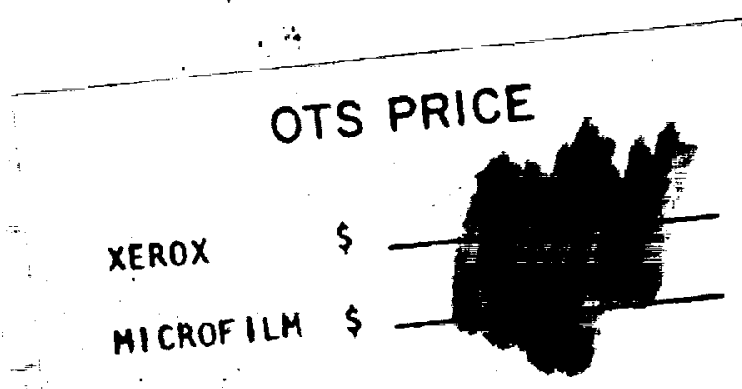

$\because$

1. - - - - - - - - 


\section{ABSTRACT}

This paper considers problems which accompany thermionic emission of electrons from a hot body surrounded by a plasma. In the absence of other mechanisms, an electric potential is established at the surface of the body through the balance of. thermionic emission and accretion of electrons from the external plasma. Analytical solutions are obtained for the electric potential field and the electron density distribution around the body. A possible application of this analysis to objects in space is indicated. 


\section{INTRODUCTION}

An object in space may become hot while approaching a hot stellar body like the sun, or while entering a dense atmosphere like that of the earth. Long before such metallic objects melt, evaporate, or ablate, they may acquire temperatures which are sufficient to cause a copious emission of electrons . from the surface. Therefore, the temperatures.lower than, and in the neighborhood of, the melting point are of interest to us in this paper. As a matter of convenience and without serious loss of generality, we will regard iron as a reference substance composing the objects in space, and hence consider temperatures lower than $1600^{\circ} \mathrm{K}$. The analytical formulae are, however, applicable to any other specific case of a surface capable of thermionic emission.

Thermionic emission is very sensitive to temperatures; the emitted electron flux is of the order of $10^{12}$ and $10^{18^{\circ}}$ electrons $/ \mathrm{cm}^{2}-\mathrm{sec}$ at surface temperatures of $1000^{\circ} \mathrm{K}$ and $1600^{\circ} \mathrm{K}$ respectively, from a material of work function $w_{0}=3 \mathrm{ev}$. The emission of electrons from the object's surface leaves a positive surface charge. A great majority of the emitted electrons describe ballistic orbits and return to the surface, while a certain number of those in the high energy tail of the energy spectrum are able to escape from the potential field of the 
object. The positive charge at the object's surface is established by these escaping electrons, and the rate of elscape of tho emitited electrone decreamos with an inerease in the surface potential. Furthermore, if the object is surrounded by a plasma, the plasma accretion alone has a tendency to impart a negative charge to the object's surface. Therefore, a steady potential can be established at the object's surface when the net negative charge leaving the object due to thermionic emission is completely replenished by the net negative charge brought to the surface by the accretion from the surrounding plasma. The magnitude of the equilibrium surface potential is then determined from the balance of the plasma accretion current and the escape component of the thermionic emission current.

There are other mechanisms (Chopra 1961) in which an object may acquire an electric charge. An effect of considerable interest is connected with the photoelectric emission and accretion of electrons. The photoelectric effect is important for objects on the day side of the earth and for surfaces exposed to the sun. In certain cases, it is comparable to, and at times may even become. more significant than, the thermionic emission. We will, however, limit the analysis of the present paper to only thermionic emission and leave these other considerations for a subsequent paper. The incoming plasma electron flux and the thermionic electrons 
constitute a plasma cloud with most of the contribution to the electron density in the cloud coming from the ballistic component of the emitted electrons. This plasma cloud screens the electric potential on the body. An analytical expression for the density distribution in terms of potential $\varphi_{0}$ and work function $w_{0}$ is obtained by solving the equations of Poisson and the conservation of energy and momentum. This analytical expression is substituted back in the poisson equation which is then solved numerically to yield potential distribution as a function of distance from the surface.

F It may be mentioned here that the problem considered in this paper bears a certain analogy to the problem of the exosphere. In the exosphere problem, the particles are projected outwards corresponding to the temperature of the base layer. One of us (C.s. Shen) has successfully applied (shen 1963) the present analysis (after some modifications) to the structure of the planetary exosphere, and has obtained an analytical expression for the density distribution.

\section{FORMULATION OF THE PROBIEM - BASIC EQUATIONS}

Let us consider a spherical object with an equilibrium surface poteritial $\varrho_{0}$ and surface temperature $T$, surrounded by 
screening charges due to thermionic emission and a rarefied external plasma with electron density $n_{e}$ and ion density $n_{e} / z$ (where $z e$ is the ionic charge at a temperature $T_{p}$. When the themionic emission is stronger than the plasma accretion and the object is moving slower than the mean thermal speed of the plasma electrons $\left(10^{7} \mathrm{~cm} / \mathrm{sec}\right)$ the potential $n(r)$ and the screening electron density $\rho(x)$ are, to a first degree of approximation, spherically symmetrical, and are given by

$$
\nabla^{2} \varphi(r)=-\frac{\mathrm{e}}{\epsilon_{0}} \rho(r)
$$

where $e=4.8 \times 10^{-10}$, e.s.u. is the electron charge, $\epsilon_{0}=1$ is the permitivity of the medium, and $r$ is the radial distance measured from the center of the spherical body. The screening electron density $\rho(x)$ consists of three parts.

$$
\rho(r)=\rho_{b}(r)+\rho_{\text {esc }}(r)+\rho_{p}(r) .
$$

Here $\rho_{b}(r)$ is the ballistic component which is due to the electrons emitted from the surface with velocities less than the escape velocity; these particles describe ballistic orbits in the electric potential field of the body and return to the surface. The escape component $\rho_{\text {esc }}(r)$ is due to the electrons emitted with velocities 
exceeding the escape velocity; these particles do not return to the charged body. The third component $p(r)$ is due to the accretion from the surrounding plasma. Among these $\rho_{b}(x)$ contributes about 90 percent to the local electron density (as can be seen from later calculations). Also, in the steady state condition, the escape component of the thermionic electron flux is equal to the incoming plasma accretion flux. Therefore, to simplify one of our later calculations, we can set $\rho_{\text {esc }}(r)=\rho_{p}(r)$. Assuming that the electrons inside the metallic body have velocities given by the Fermi distribution law, the number of electrons having velocities in the range $(\vec{v}, \vec{v}+d \vec{v})$ and hitting a unit area of the surface (inside) is given by

$$
J(\vec{v})=\frac{4 \pi m^{3} v_{x} v_{t} d v_{r} d v_{t}}{h^{3} e^{\left(E-E_{f}\right) / k T+1}}
$$

where

$$
E=1 / 2 m\left(v_{r}^{2}+v_{t}^{2}\right)
$$

and $\mathrm{m}=9 \times 10^{-28} \mathrm{~g}$ is the electron mass, $\mathrm{h}=6.27 \times 10^{-27} \mathrm{erg} / \mathrm{sec}$ is the Planck's constant, $E_{E}$ is the Fermi energy, and $v_{r}$ and $v_{t}$ are the components of the velocity $\vec{v}$ in directions parallel and transverse to the radius vector $\vec{r}$.

If we denote the velocity of the electron at the position 
$\vec{r},(x>R)$, by $\vec{u}(x)$, then the principles of conservation of energy and angular momentum require that

$$
r u_{t}=R v_{t}
$$

and

$$
1 / 2 m\left(u_{x}{ }^{2}+u_{t}{ }^{2}\right)-e \varphi(r)=1 / 2 m\left(v_{x}{ }^{2}+v_{t}^{2}\right)-w_{0}-E_{f}-e n_{0}(r)
$$

where $R$ is the radius of the body.

$$
\text { Equations (4) and (5) yield }
$$

$$
u_{r}^{2}=v_{r}^{2}+\left(I-\frac{R^{2}}{r}\right) v_{t}^{2}-\frac{2}{m}\left\{e\left(\varphi_{0}-(n)+E_{f}+w_{O}\right\}\right.
$$

which provides a stringent condition for an electron emitted from the surface to reach the radial distance $r$. Only those electrons with initial velocity $\vec{v}$. satisfying the inequality

$$
v_{r}^{2}+\left(1-\frac{R^{2}}{r^{2}}\right) v_{t}^{2}-\frac{2}{m}\left\{e\left(n_{0}-\omega\right)+E_{f}+w_{0}\right\} \geq 0
$$

can reach position $r$. These electrons may be divided into two categories:

1) Ballistic component: These electrons satisfy Equation (7) and have velocities less than the velocity of escape such that

$$
1 / 2 m v^{2}-W_{0}-E_{f}<e \varphi_{0}
$$


and hence describe ballistic orbits.

2) Escape Component: These electrons satisfy Equation (7) and have velocities equal to or exceeding the velocity of escape such that

$$
1 / 2 m v^{2}-w_{0}-E_{f} \geq e_{0}
$$

and describe escape trajectories.

These classifications are important in the evaluation of electron density and may be illustrated diagrammatically as in Figure 2. Curves I, II, and III describe equations

$$
\begin{gathered}
v_{r}^{2}+v_{t}^{2}=\left(\frac{2}{m}\right)\left[E_{f}+w_{0}+e \varphi_{0}\right] \\
v_{r}^{2}+\left(1-\alpha^{2}\right) v_{t}^{2}=\left(\frac{2}{m}\right)\left[E_{f}+w_{0}+e\left\{\varphi_{0}-\varphi\right\}\right],
\end{gathered}
$$

and

$$
v_{r}^{2}=\left(\frac{2}{m}\right)\left[E_{f}+w_{0}\right]
$$

where $\alpha=R / r$. These curves represent a circle, an eliipse and a straight line in the same order and distribute the thermionic electrons in various velocity domakins.

The electrons with velocity domains external to the circle and the straight line - region $\mathrm{A}$ - are the escape electrons which do not return to, the body. The electrons with velocities 
in the domain enclosed by the circle and the ellipse - region $B$ belong to the ballistic group with more than the necessary radial component of the velocity to reach the position $r$. These electrons are counted twice in calculating the electron density distribution and make a dominant contribution to the local electron population. The electrons corresponding to region $c$ - enclosed by the ellipse and the straight line - also belong to the ballistic group but do not possess enough radial velocity to reach position $r$. Therefore, these particles do not contribute to the local electron density. The straight line represents the least value of the radial velocity that an electron must acquire before it can surmount the surface barrier. Therefore, the electrons corresponding to region $D^{\prime}$ in Figure 1 are not able to get out of the surface of the metallic body.

\section{ELECTRON DENSITY AS A FUNCTION OF POTENTIAL}

The contribution of the thermionic electrons with the initial (just inside the surface) velocities in the range $(\vec{v}, \vec{v}+d \vec{v})$ to the electron population in a shell of radii $r$ and $r+d r$ is determined by the product of the corresponding electron flux $J(\vec{v}) d \vec{v}$ and the time $d t=d r / u_{r}$ spent by these electrons in traversing the thickness dr of the shell. This contribution $d \rho_{t h}(r)$ is given by. 


$$
d \rho_{\operatorname{th}}(r)=\sigma^{2} \int_{u_{r}>0} \frac{J(\vec{v}) d \vec{v}}{u_{r}}
$$

which with the help of Equations (3) and (6) yields the expression for the thermionic component $p_{t h}(r)$ of the electron density at position $r$,

$$
\rho_{t h}(r)=4 \dot{\pi}\left(\frac{m^{3}}{h}\right) a^{2}\left[2 I_{1}+I_{2}\right]
$$

where

$$
\begin{aligned}
& -I_{1}=\int_{1} \int \frac{v_{x} v_{t} d v_{r} d v_{t}}{\left[v_{x}{ }^{2}+\left(1-\alpha^{2}\right) v_{t}{ }^{2}-(2 / m)\left\{E_{f}+w_{0}+e\left(\varphi_{0}-\varphi\right)\right\}\right]^{1 / 2}\left[e^{\left.\left[m\left(v_{r}{ }^{2}+v_{t}{ }^{2}\right)-2 E_{f}\right\} \cdot \mid 2 k T_{+1}\right]}\right.} \\
& \quad \text { Region } B \\
& \quad \text { and. }
\end{aligned}
$$

$\because I_{2}=\iint \frac{v_{r} v_{t} d v_{r} d v_{t}}{\left[v_{r}{ }^{2}+\left(I-\alpha^{2}\right) v_{t}{ }^{2}-(2 / m)\left\{E_{f}+w_{0}+e\left(\omega_{0}-\varphi\right)\right\}\right]^{1 / 2}\left[e^{\left.\left\{m\left(v_{r}{ }^{2+v_{t}}{ }^{2}\right)-2 E_{f}\right\} / 2 k_{s}{ }^{T}+1\right]}\right.}$

Region $\mathrm{A}$

and the limits of the integrals $I_{2}$ and $I_{2}$ are set in accordance with Equations (10) - (12) and Figure 1, and the weight factors are inserted as explained in the preceding section. Including the contribution of the extemal plasma, the total electron density $o(x)$ 
at the position $x$ becomes

$$
\rho(r)=\rho_{t h}(r)+\rho_{p}(r)=8 \pi(m / h)^{3} \alpha^{2}\left(I_{1}+I_{2}\right) .
$$

On introducing the following dimensionkess parameters

$$
\mathrm{x}^{2}=m v_{r}^{2} / 2 \mathrm{kT} ; \mathrm{Y}^{2}=\mathrm{mv}_{t}^{2} / 2 \mathrm{kT} ; \varepsilon=\mathrm{E}_{\mathrm{f}} / \mathrm{kT}
$$

and

$$
a=\left[E_{f}+w_{0}+e\left(\omega_{0}-\varphi\right)\right] / k T,
$$

Equation (17) reduces to

$$
\begin{gathered}
\rho(x)=\frac{8 \pi(2 m k T)^{3 / 2} \alpha^{2}}{h^{3}} \iint \frac{X X d X d Y}{\left[X^{2}+\left(1-\alpha^{2}\right) Y^{2}-a\right]^{1 / 2}\left[e^{X^{2}+Y^{2}-\epsilon}+1\right]} \\
X^{2}+\left(1-a^{2}\right) Y^{2}-a \geq 0
\end{gathered}
$$

But,

$$
x^{2}+y^{2}-\epsilon \geq a-\epsilon \geq w_{0} / K T>1
$$

and, therefore, we can neglect the unity term in comparison with the exponential term in the denominator of Equation (19). Furthermore, on setting

$$
x^{2}+\left(1-\alpha^{2}\right) y^{2}-\epsilon-\left(w_{0} / k T\right)=z^{2}
$$


and

$$
\left(1-a^{2}\right)^{1 / 2}(\mathrm{Y} / \mathrm{X})=\tan R
$$

in the last equation, and after some simplification, the expression for the total electron density $p(r)$ reduces to

$$
\begin{aligned}
& \rho(x)=2\left(2 \pi m k T / r^{2}\right)^{3 / 2} e^{-\left\{w_{0}+e\left(m_{0}-\varphi\right)\right\} / k T} \\
& {\left[1-\left\{1-(R / r)^{2}\right\}^{1 / 2} e^{-R^{2} e\left(\omega_{0}-\varphi\right) / k T\left(r^{2}-R^{2}\right)}\right]}
\end{aligned}
$$

which is expressed as a function of the potential $\varphi(r)=\varphi$.

IV. REDUCED POISSON EQUATION

Substituting for the electron density $p(r)$ from Equation

in the poisson Equation ( 1 ) and introducing the dimensionless quantities

$$
\psi=e \omega / k T
$$

and

$$
\theta=W_{0} / K T \quad,
$$

we obtain

$\left.\frac{1}{r^{2}} \frac{d}{d r}\left(r^{2 d} \frac{d r}{d r}\right)=A(k r)\right)^{1 / 2} e^{-\left(\psi_{0}-\psi\right)}\left[1-\left\{1-(R / r)^{2}\right\}^{I / 2} e^{-\left(\psi_{0}-\psi\right) R^{2} /\left(r^{2}-R^{2}\right)}\right]$ 
where

$$
A=-2\left(e^{2} / \varepsilon_{0}\right)\left(2 \pi m / h^{2}\right)^{3 / 2} e^{-\theta}
$$

and the boundary conditions of the problem are

$$
\psi(R)=\psi_{0}
$$

at $r=R$, and

$$
\psi(\infty)=0
$$

at $r=\infty$.

In some cases of interest to us, we will find that the equilibrium potential energy $e \varphi_{o}$ is much greater than the thermal energy $\mathrm{KT}$ corresponding to the surface temperature $\mathrm{T}$. This would then enable us to neglect, to a first approximation, the second term in Equation (24), and hence we have

$$
\nabla^{2} \psi(x)=B e^{-X}
$$

with

$$
B=A(k T)^{I / 2} \text {, and } X=\psi_{0}-\psi \text {. }
$$

Equation (27) is identical to the so-called isothermal equation which has been solved for various boundary conditions and applied extensively to the problems pertaining to stellar structure by Chararasekhar (1939). . 


\section{DETERMINATION OF SURFACE POTENTIAL}

The equilibrium value of the surface potential is detemined Erom the balance of the escape component of the thermionic emission current and the plasma accretion current. The plasma accretion current consists of the electron and ion components. In the absence of a surface potential $\varphi_{0}$, the ion accretion current is smaller than, the electron accretion current by a factor of the order of $\left(m_{e} / m_{i}\right)^{1 / 2}$. Therefore, only the relative initial magnitudes of the thermionic escape current and the plasma electron accretion current need be considered, and the ion accretion current may be neglected. Then, the surface potential $\varphi_{0}$ is positive if the initial thermionic escape current is greater than the initial electron accretion current. It may, however, become necessary to include ion accretion in consideration of the magnitude of the surface potential $\omega_{0}$, if the latter is negative.

Let us first consider the case of a positive surface potential. Then, the themionic escape current is given by

$$
J_{e s c}=8 \pi R^{2}(m / h)^{3} \iint \frac{v_{r} v_{t} d v_{r} d v_{t}}{e^{-\left\{m\left(v_{x}{ }^{2}+v_{t}{ }^{2}\right)-2 E_{f}\right\} / 2 k T+1}}
$$

Region $\mathrm{A}$ 
As mentioned in section II, the electrons in region $A$ of Figure 1 must have radial and transverse velocity components such that

$$
v_{r}^{2}>(2 / m)\left(E_{E}+w_{0}\right)
$$

and

$$
v_{r}^{2}+v_{t}^{2}>(2 / m)\left(E_{f}+w_{0}+e \varphi_{0}\right)
$$

Therefore, the expression for the thermionic escape component may be rewritten as

$$
\begin{aligned}
& \sqrt{(2 / m)\left(E_{f}+w_{0}+e n_{0}\right)} \\
& J_{\text {esc }}=8 \pi R^{2}(\mathrm{~m} / \mathrm{h})^{3}\left[\int v_{x} d v_{r} \int^{\infty} \frac{v_{t} d v_{t}}{e^{-\left\{m\left(v_{r}{ }^{2}+v_{t}{ }^{2}\right)-2 E_{f}\right\} / 2 k T}+1}\right. \\
& \left.\sqrt{(2 / m)\left(E_{f}+w_{0}\right.}\right) \quad \sqrt{(2 / m)\left(E_{f}+w_{0}+e \varphi_{0}\right)} \\
& \left.+\int^{\infty} v_{r} d v_{r} \int_{0}^{\infty} \frac{\dot{v} d v t}{e^{-\left\{m\left(v_{r}{ }^{2}+v_{t}^{2}\right)-2 E_{f}\right\} / 2 k T+1}}\right] \\
& \sqrt{(2 / m) \cdot\left(E_{I}+W_{O}+e \varphi_{0}\right)}
\end{aligned}
$$


Once again we can neglect the unity terms in comparison with the exponential terms in the denominators of the integrands in Equation (31). After carrying out the integration and making some simplifications, Equation (31) reduces to

$$
J_{\text {esc }}=(4 \pi R k T)^{2}\left(\mathrm{~m} / \mathrm{h}^{3}\right)(I+\psi) e^{-\left(\psi_{0}+\theta\right)}
$$

For bodies moving slower than the mean thermal speed of the plasma electrons, the electron accretion is symmetrical about the body, and is given by

$$
J_{e}=\frac{2 \pi}{3} \eta_{e} n_{e} v_{e} R^{2} e^{\left(T / T_{p}\right) \psi_{0}}
$$

when $n_{e}$ and $v_{e}$ are the number density and the mean thermal speed of the plasma electrons, and $\eta_{e}$ is the sticking coefficient defined as the fraction of the incident electrons transferring their charge to the body. In estimating the plasma accretion current $J_{p}$ we further note that the ion accretion is further reduced by a factor $e^{-\left(T / T_{p}\right)} \psi_{0}$ and becomes negligibly small as compared to the electron accretion. Hence,

$$
J_{p} \cong J_{e}=\frac{2 \pi}{3} \eta_{e} e_{e} e^{R^{2}} e^{\left(T / T_{p}\right)} \psi_{0}
$$

which, when combined with Equation (32) in the condition of equisibrium, 


$$
\begin{gathered}
-18- \\
J_{\text {esc }}=J_{p},
\end{gathered}
$$

yields

$$
\frac{e^{\left[I+T / T_{p}\right] \psi_{0}}}{I+\psi_{0}}=\frac{24 \pi m_{e}(k T)^{2} e^{-\theta}}{\eta_{e} n_{e} e^{h^{3}}}=6 \times 10^{29} \frac{T^{2} e^{-\theta}}{T_{n_{e}} v_{e}} \text {, }
$$

where $I$ is expressed in electron volts.

IF; on the other hand, the surface potential $\varphi_{0}$ is negative, the ion accretion current is enhanced by a factor of $e^{\prime\left(T / T_{p}\right)} \psi_{0} l$. With Wo $_{0}$ larger than a few tenths of an electron volt, the enhancement factor el $\psi_{0} /$ may be large enough to counteract the " effect of the reduction factor $\left(m_{e} / m_{i}\right)^{1 / 2}$ so that the ion current may by no means be negligible. In these circumstances, we must include the term

$$
J_{i}=(2 \pi / 3) \eta_{i} z_{i} n_{i} v_{i} R^{2} e^{-\left(T / T_{p}\right) \psi_{0}}
$$

in calculation of $\mathrm{J}_{\mathrm{p}^{-}}$. In writing Equation (37) we have assumed that the ion accretion is also symmetrical about the body. If, . however, the speed of the body exceeds the mean thermal speed of the plasma ions by an order of magnitude, the ion accretion current (Equation (37)) is reduced by a factor of $1 / 2$. The corresponding electron accretion current is given by 


$$
J_{e}=(2 \pi / 3) \eta_{e} n_{e} v_{e} R^{2} e^{\left(T / T_{p}\right)} \psi_{0} .
$$

Fherefore, the expression for the plasma accretion current. reduces to

$$
J_{p}=J_{e}-J_{i}=(2 \pi / 3) R^{2} n_{e} v_{e}\left[\eta_{e} e^{\left(T / r_{p}\right)} \psi_{o-\eta_{i}}\left(m_{e} / m_{j}\right)^{1 / 2} e^{-\left(T / T_{p}\right) \psi_{0}}\right]-
$$

In the calculation of the thermionic escape current we mav first remark that the negative surface potential in our problem is only a fraction of a volt. It may also be noted that a negative surface potential, however small, enables all the emitted electrons to escape. Hence, the thermionic escape current is approximately given by

$$
J_{e s c}=(4 \pi R k T)^{2}\left(m / h^{3}\right) e^{-A} .
$$

Finally, in the condition of equilibrium (Equation (35)) Equations (39) and (40) yield

$$
\begin{gathered}
\eta_{e} e^{\left(T / T_{p}\right)} \psi_{0}-\eta_{i}\left(m_{e} / m_{i}\right) I / 2 e^{-\left(T / T_{p}\right)} \psi_{0}=24 \pi\left(m_{e} / h^{3} n_{e} v_{e}\right)(k T)^{2} e^{-\theta} \\
\simeq 6 \times 10^{29}\left(T^{2} / n_{e} v_{e}\right) e^{-\theta}
\end{gathered}
$$




\section{DISCUSSION}

In the preceaing sections, we have formulated and analyzed the pioblem of the screening of the electric potential on a hot spherical ojject surrounded by an extemal plasma. It is assumed that i) the spherical body acquires the electric potential in the processes of the themionic emission of electrons from the surface. of the object and the accretion of the charged particles from the surrounding plasma, and 2) the surface potential and the distribution of the potential and the electron density in the screening cloud are spherically symetrical about the object. The basic requirement to satisfy these assumptions are that i) the surface of the spherical object is at a uniform temperature and ii) the object is either at rest or it moves with a speed that is small compared to the mean themal speed of the plasma electrons. These recuirements set restrictions on the exact application of the results of the present analysis to actual objects in space. The present analysis, nevertheless, provides, even in such cases where the above-mentioned assumptions do not strictly hold, at least an oràer of magnitude estimate of this phenomenon in front of the hottest part of the object. The applications of our analysis may be found in objects $\therefore$ 
entering a planctary atmosphere or those approaching sufficiently close to a hot star. A space vehicle entering the earth's armosphere encounters stagnation temperatures of the order of $1500^{\circ} \mathrm{K}$. 2.2 meteoric objects accuire surface temperatures above $1200^{\circ} \mathrm{K}$. Ionization in front of the cometary heads and certain cometary tails which is not understood as well, may be attributed in part to the nolar heating of the metallic content of these objects. In general, the surface temperatures of the above-mentioned classes of objects are not uniform. Due to the variety of the types of such objects and uncertain available data, we will not make any attempt to apply our analysis to any specific case of the abovementionec space objects. Instead, we will illustrate our theory by consicering a hypothetical spherical object heated to a uniform surface temperature and surrounded by a plasma of electron density $\mathrm{n}_{\mathrm{e}} \sim 10^{3} / \mathrm{cm}^{3}$ at the equilibrium plasma temperature $\mathrm{T}_{\mathrm{p}} \sim 1000^{\circ} \mathrm{K}(0.09 \mathrm{ev})$ Two vilues of the work function and five values of the surface temperature, viz..

$$
W_{0}=3.0 \text { and } 3.8 \text { (electron volts) }
$$

and

$$
T=0.0 \hat{x}, 0.06,0.09,0.13 \text { and } 0.15 \text { (electron volts) }
$$

are considered to illustrate tho influence of these parameters 


$$
-22-
$$

on the nature of the electron cloud around a hot object. A common value of $\eta_{e}=\eta_{i}=0.1$ is adopted for the sticking cosficients. Since these surface-plasma parameters appear in a logarithnic term, any departure from this value for the sticking coefficients is not likely to seriously affect our result.

Ta. aulibrium value of the surface potential $\omega_{0}$ is determined by the surface temperature $T$, the electron density $n_{e}$, and temperature $T_{p}$ of the surrounding plasma. At low values of $T$, themionic emission of electrons is small, and hence, the balance of the electron and ion accretion currents from the surrounding plasma establishes a negative potential $\varphi_{0}$ on the object's surface. The numerical value of $\phi_{0}$ is always a fraction (s 0.05 ) of a volt because even this small value of $\varphi_{0}$ is large enougin to increase substantially the ion accretion current and reduce the electron accretion current to off-set the relative effect of the factor $\left(m_{e} / m_{i}\right)^{1 / 2}$.

\section{Table 1}

SUREACE POTENTIAL OF AVMEMALLIC BODY

\begin{tabular}{lll}
\hline$W_{0}=3.8 \mathrm{ev} \quad \mathrm{T}_{\mathrm{p}}=0.09 \mathrm{ev}\left(1044^{\circ} \mathrm{K}\right)$ & $n_{\mathrm{e}}=10^{3} / \mathrm{c.c} \quad \eta=0.1$ \\
\hline $\mathrm{T}(\mathrm{ev})$ & $n_{0}(\mathrm{volt})$ \\
0.04 & -0.1691 & \\
0.06 & -0.1683 & \\
0.09 & +0.0963 \\
0.11 & +0.4895 \\
0.13 & +0.8430 \\
0.15 & +1.1340
\end{tabular}


At high values of $\mathrm{i}$, on the other hand, a positive surface potential of several volts is established by the balance of the themionic-emission and the elcctron-acoretion currents: the ion-acoretion current having been reduced to a negligible value by the joint action of the positive potential and the factor $\left(m_{e} / m_{i}\right)^{1 / 2}$. Table 1 lists values of $\varphi_{0}$ corresponding to the several values of $W_{0}$ and $T$.

Equation (24) can be reduced to a dimensionless differential equation.

$$
\frac{I}{x^{2}} \frac{d}{d x}\left(x^{2} \frac{d \psi}{d x}\right)=A R^{2}(K T)^{i / 2} e^{-\left(\psi_{0}-\psi\right)}\left[I-\left(1-x^{-2}\right)^{2}\right]^{1 / 2} e^{-\left(\psi_{0}-\psi\right) /\left(x^{2}-1\right)}
$$

where $x \cong \frac{r}{R}$. The variation of potential with distance from the sprerical hot body of l-cm radius is calculated by solving Equation (42), and the results are illustrated in Figures 2 and 3. The two curves representing the inclusion and the exclusion of the secona term inside the parenthesis of Equation (42) for the set of parameters $T=0.09 \mathrm{ev}, w_{0}=3 \mathrm{ev}$ and $\psi_{0}=5.43$ are show in Figure 2, while Eigure 3 exhibits the profile of the potential distribution, with the inclusion of the second term in Equation in the numerical calculations, for the two sets of parameters:

$$
\text { i) } T=0.13 \mathrm{evi} w_{0}=3.8 \mathrm{ev} ; \text { and } \psi_{0}=6.5 \text {, }
$$


ana

$$
\text { : ii) } T=0.11 \mathrm{ev} ; w_{0}=3.8 \mathrm{ev} ; \text { and } \psi_{0}=4.0
$$

respectively. The variation of potential with distance has the Eollowing characteristics:

1) The nature of the profile of the potential aistribution. curve is independent of the set of the parameters used. The potential falls very rapidily with distance from the object. and reduces to $1 / 3$ of its surface value at a distance of approximately 2.3 and $1.7 \mathrm{~cm}$ in Figures 2 and 3 respectively. At a cistance of about $8-10 \mathrm{~cm}$ the potential acquires an almost zero value and the surface potential of the body is completely shielded by an electron cloud of this dimension.

2) The inclusion or the disregard of the second term inside the parenthesis of Equation (42) does not seem to matter in the calculation of the potential distribution. It is apparently due to the very rapid decrease of potential with distance from the object wich reduces this term to a second order of exponential in - $\psi_{0}$ thereby making it negligible in comparison to the first term.

The electron ciensity in the electron cloud surrounding the body is culouicted from Equation (22) by substituting in it the values of the potential distribution obtained from the solution 
of Euation (42). The results of this computation are given in Pigures 4 and 5. Figure 4, like Figure 2, includes two curves, one of these corresponds to the inclusion of the second term inside tho parentreses of Equation (22) while the other disregards this term. The set of parameters used in the computation of these curves have the value $T=0.09 \mathrm{ev} ; W_{0}=3 \mathrm{evi} \psi_{0}=5.43$. rhe des. ity distribution curve in Figure 5, as in Figure 3 , corresponds to the sets of parameters having values

$$
\text { i) } \mathrm{T}=0.09 \mathrm{ev} ; w_{0}=3 \mathrm{ev} ; w_{0}=5.43
$$

and

$$
\text { ii) } T=0.13 \mathrm{ev} ; \mathrm{w}_{0}=0.38 \mathrm{ev} ; \psi_{0}=6.5
$$

and the rumerical calculations are based on the inclusion of the second term in Equation (22). These curves bring out the Following features of the variation of the electron density with distance from the object:

1) There is a considerable increase of electron density in the immediate vicinity of the body.

2) The electron density decreases very rapialy with distance from the body.

3) Unlike in the estimates of the potential distribution, the inclusion or exclusion of the second term within the parenthesos of Equation (22) in the computations of the electron 
censity appears to make a substantial difference in these estimates (see Figure 4). The neglect of this term yields a value for the elestron censity at great distances which is highor than the ambient value. Therefore, it is necessary to consian this term in oxder to arrive at the correct estimates of the electron density.

保 Wish to thank Dr: J. Herring for helpful discussions and Dr. H. Zapolsky for his helpful comments on some mathematical aspects of the analysis.

\section{REFERENDES}

Chandrasekhar, S. 1939. An Introduction to the Study of stellar structure, (Chicago: At the University Press), IV. Chopra, K.P.. 1961, kev. vodern Phys., 33, pp. 153-189. Sher, C:S. 1963, J. Atmos. Sci. 21 . 
CARPIONS TO EIGURES

Pigure 1 - Curves I, II and III are the plots of $v_{x}$ and $v_{t}$ in cocoinande with the Equations (12), (13) and (14) respectively, anci define the velocity domajus of the themionic electrons. Electrons in domain A form the escape-group, whereas those in dom: $B$ and $C$ describe pallistic orbits with sufficient and insufficient energies respectively to reach position $r$. Eigure 2 - Plots of $\psi(r)$ against $r / R . \quad(W=3 e v ; T=0.09 \mathrm{ev}$; and $\left.\psi_{0}=5.43.\right)$ solid line $=$

$$
\nabla_{\psi}^{2}=A(K T)^{1 / 2}\left[1-\left\{1-(R / x)^{2}\right\}^{1 / 2} \exp \left(\psi-\psi_{0}\right) F^{2} /\left(x^{2}-R^{2}\right)\right] \exp \left(\psi-\psi_{0}\right) \cdot
$$

Dotted line $=\nabla^{2} \psi=A(k T)^{1 / 2} \exp \left(\psi-\psi_{0}\right)$

Eigure 3 - Solia line $=W_{0}=3.8 \mathrm{ev} ; \mathrm{T}=0.13 \mathrm{ev} ; \psi_{0}=6.50$. Dottea Iine $=W_{0}=3.8 \mathrm{ev} ; \mathrm{T}=0.1$ lev; $\dot{v}_{\mathrm{O}}=4.45$.

Figure $4-\left(W_{0}=3 e v ; r-0.09 e v ;\right.$ and $\left.\psi_{0}=5.43.\right)$ plots of $\log _{n} \rho(r)$ against $r / R$. Solja line $=$

$$
\rho=A\left[I-\left\{I-(R / x)^{2}\right\}^{1 / 2} \exp \left(\psi-\psi_{0}^{k}\right) R^{2} /\left(x^{2}-R^{2}\right)\right] \exp \left(\psi-v_{0}\right) .
$$

Dotted jine - $p=A \exp \left(\psi-\psi_{0}\right)$

zigune $5-$ Solid line $=w_{0}=3.8 \mathrm{ev} ; \mathrm{T}=0.133 \mathrm{v} ;$ and $\psi_{0}=6.50$. 2atrea $i$ ine $=W_{0}=3 e v ; T=0.09 e v ;$ and $\psi_{0}=5.43$. 


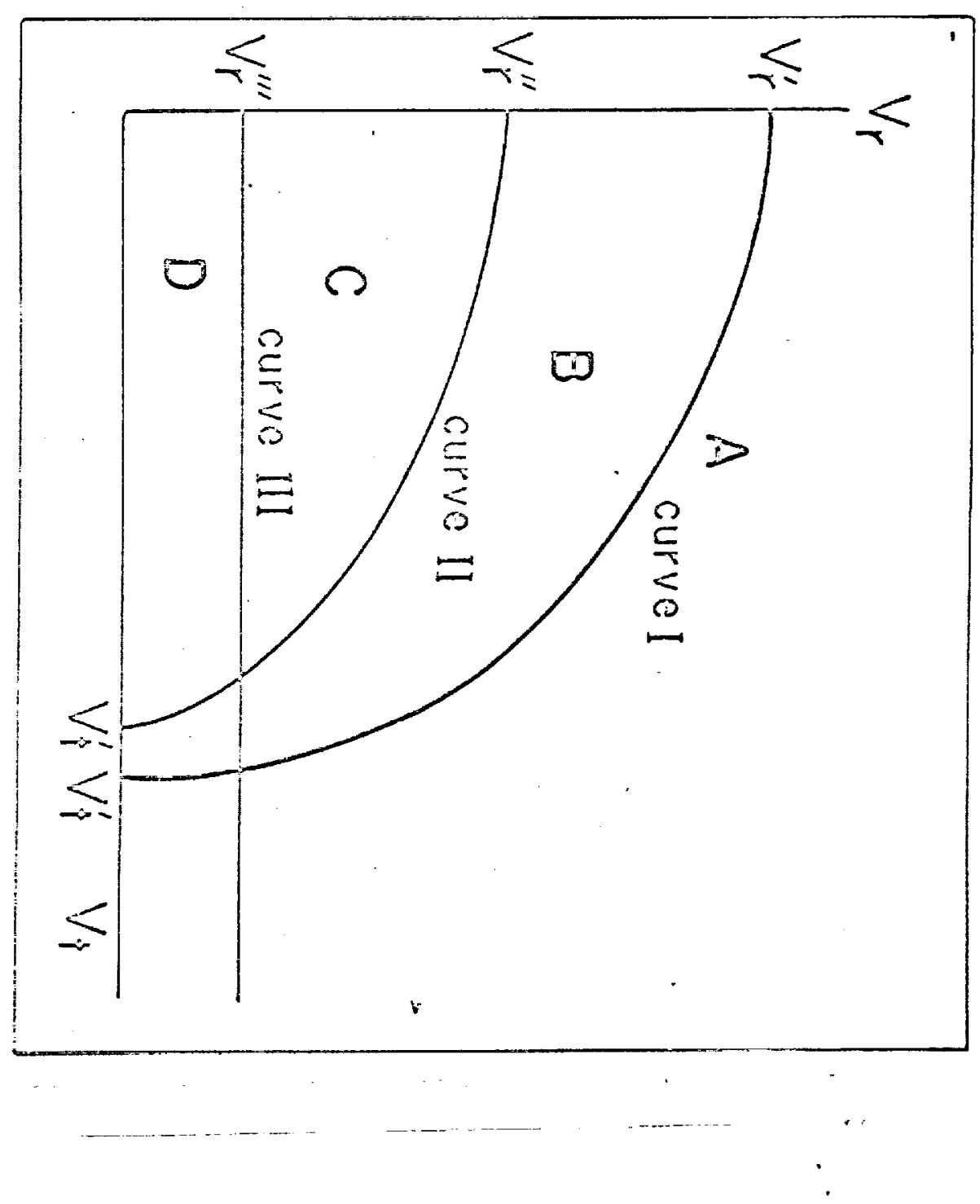




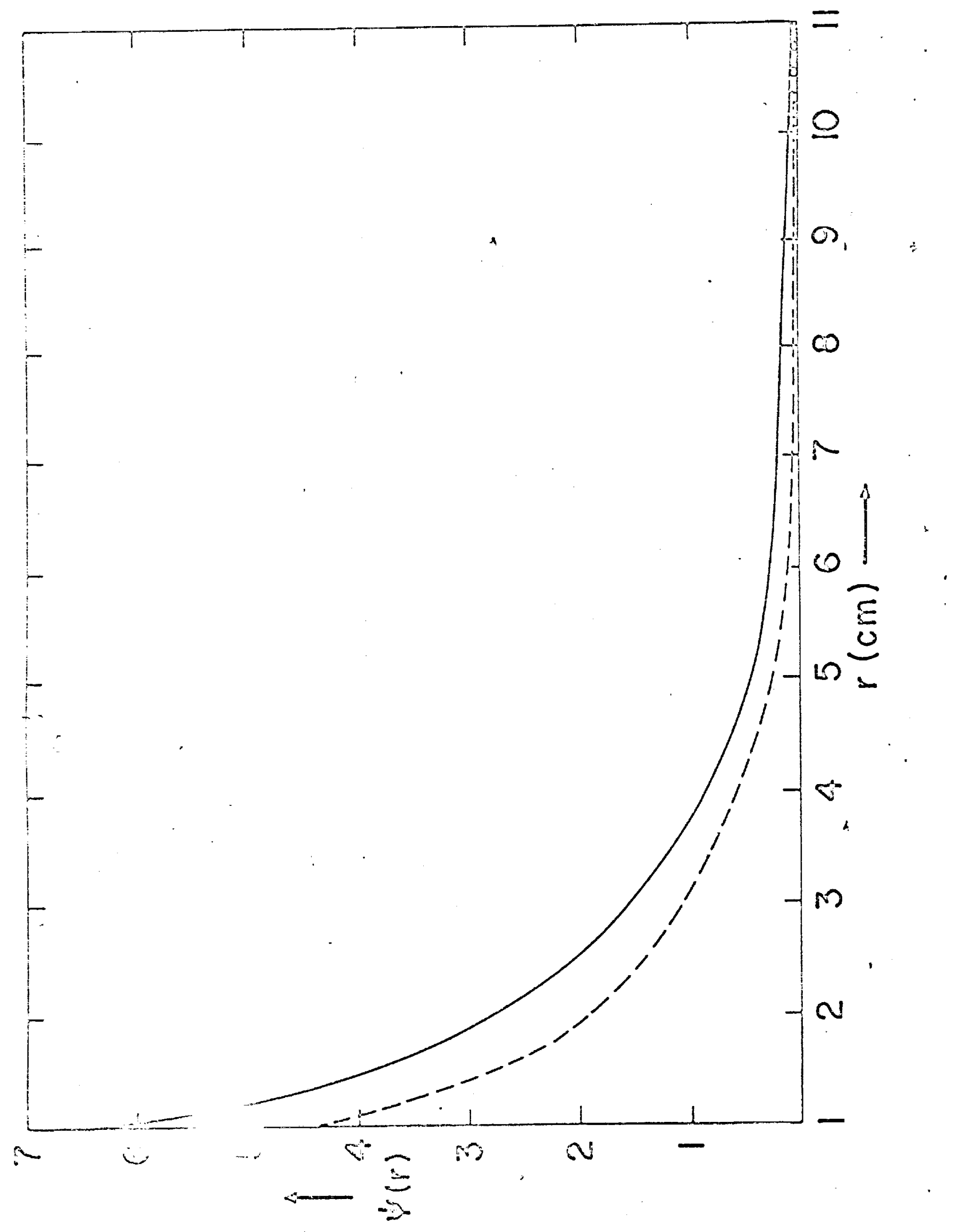




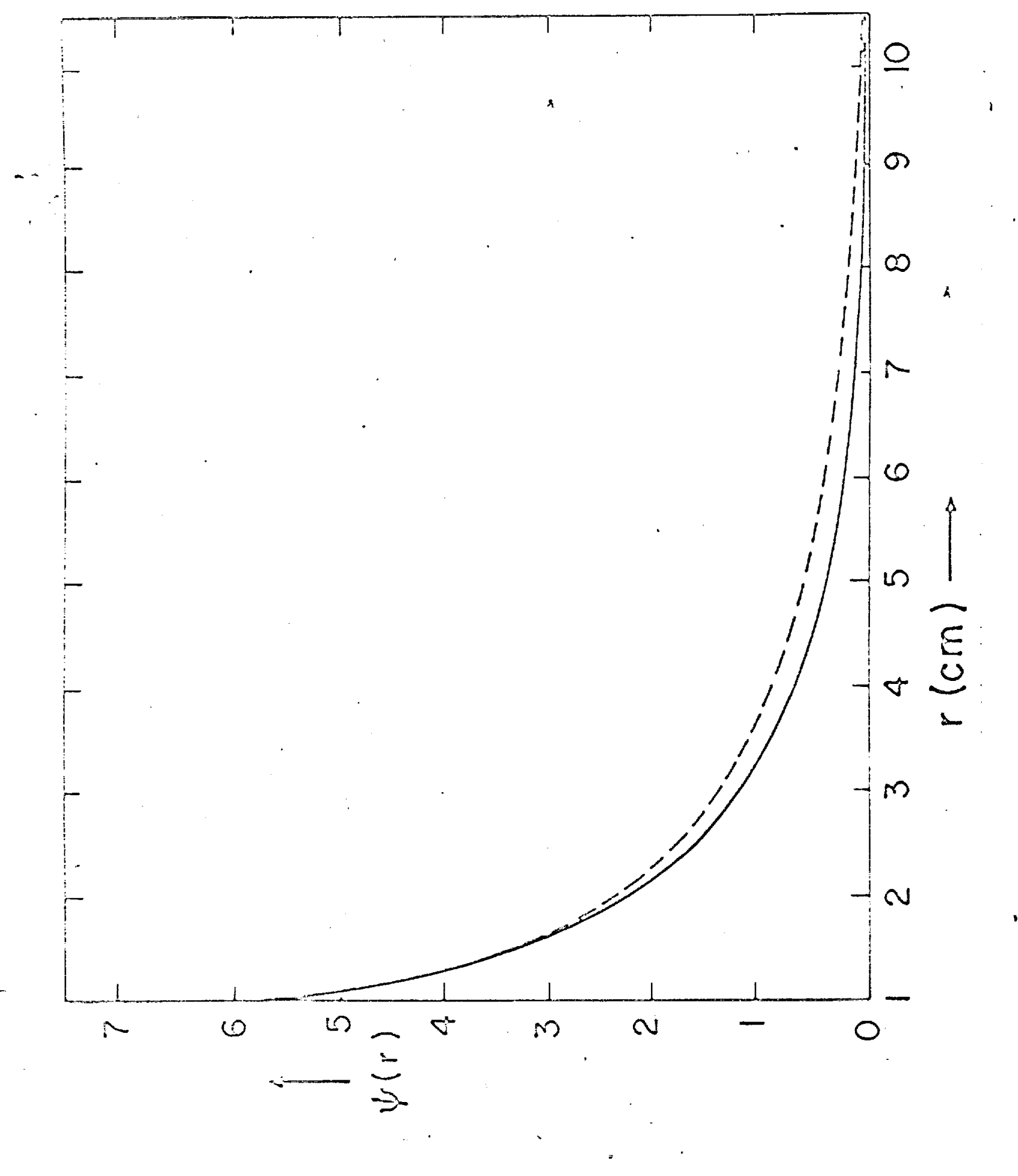




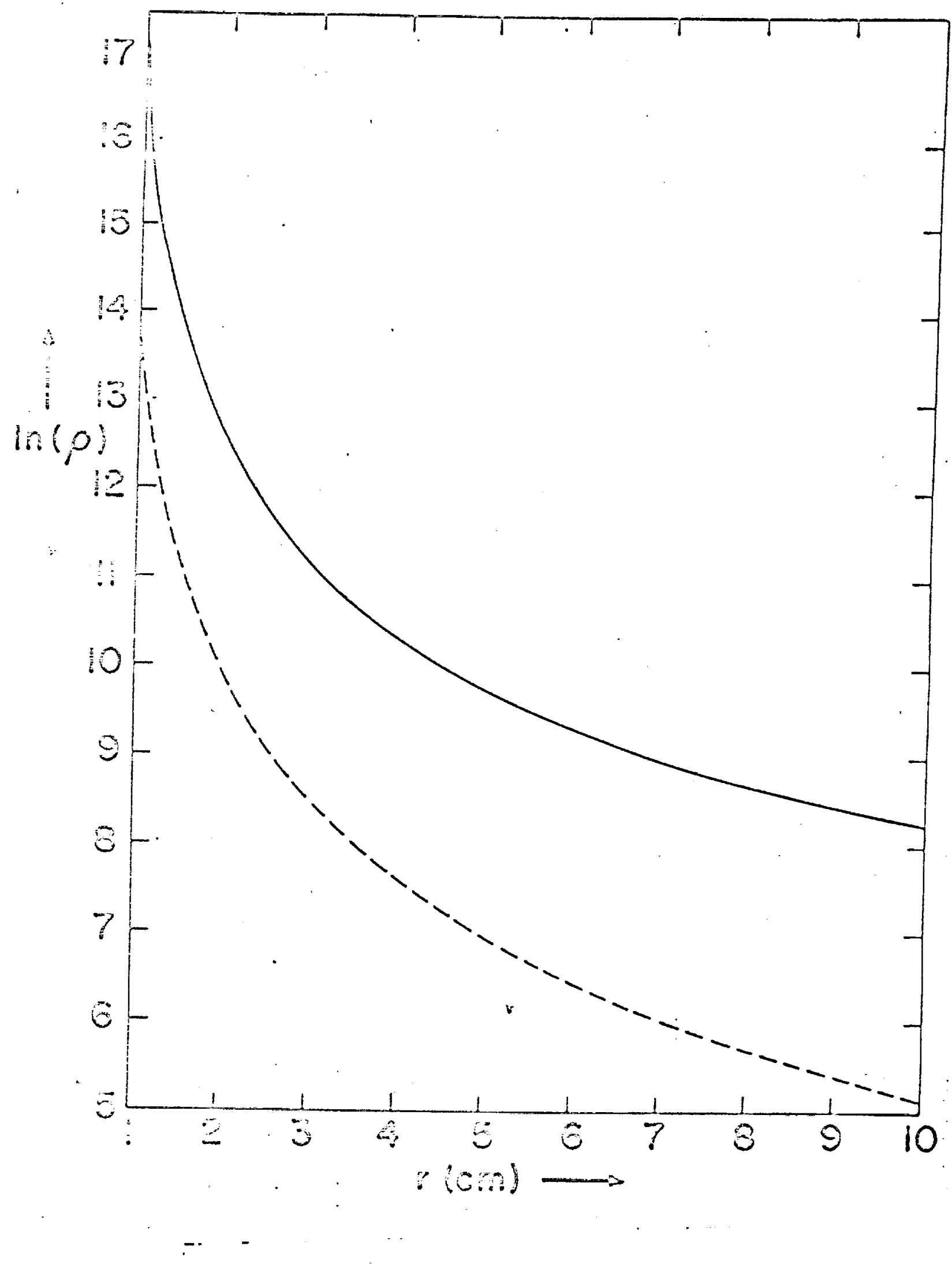




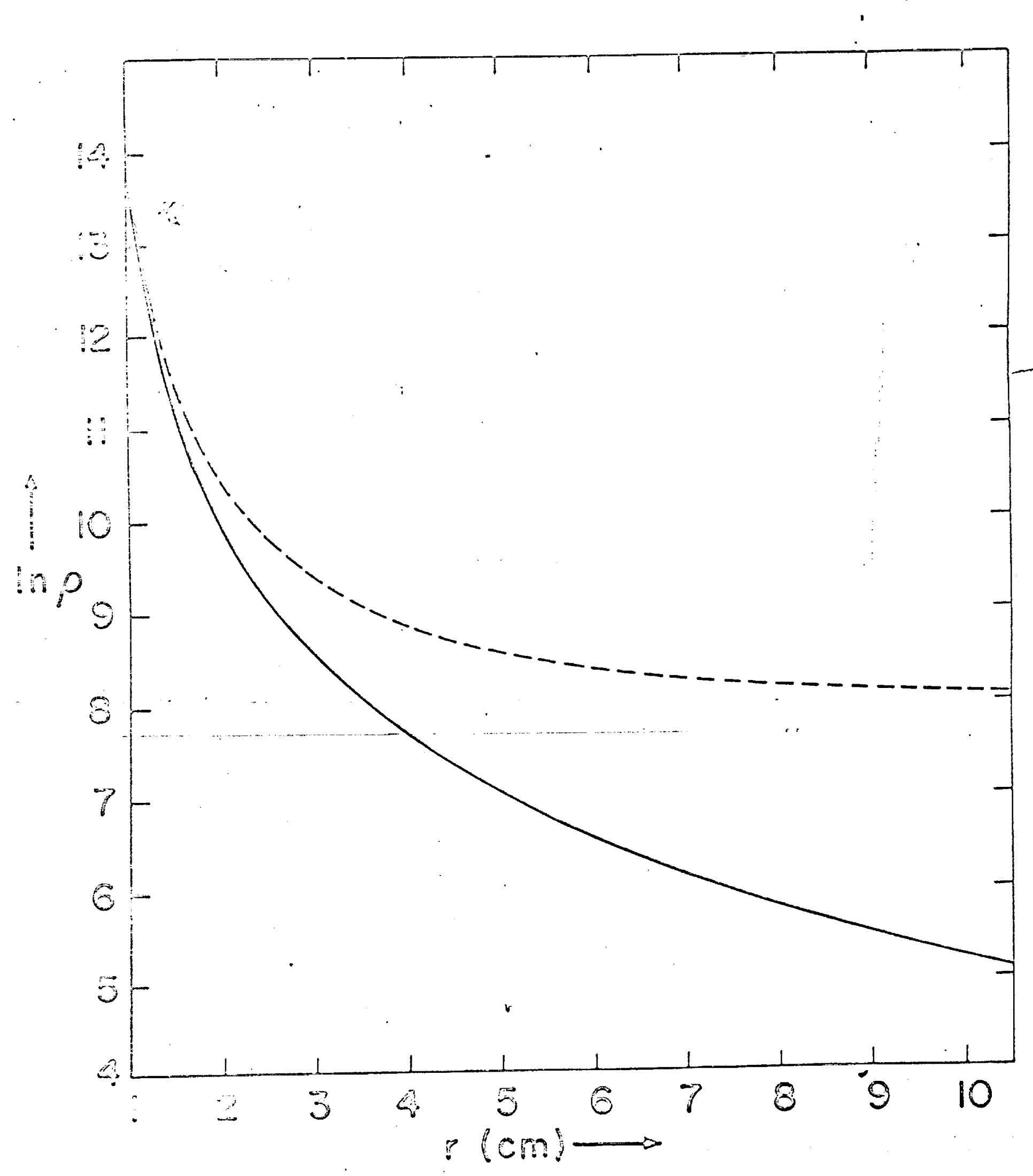

